\title{
Concepciones alternativas de alumnos de segundo y tercer ciclo de primaria, sobre el sistema Sol-Tierra-Luna
}

\section{Misconceptions of students of primary school about the Sun-Earth-Moon system}

Fátima Redondo Moralo y Florentina Cañada Cañada*

\section{RESUMEN}

En este estudio se muestran las ideas sobre el sistema Sol-Tierra-Luna que presenta un grupo de alumnos de educación primaria (8-12 años). El instrumento utilizado para recolectar los datos ha sido un cuestionario con seis preguntas abiertas. El análisis cualitativo de las respuestas reveló que un alto porcentaje de los alumnos encuestados posee concepciones alternativas, alejadas de las científicamente correctas, respecto a la órbita terrestre, las estaciones del año y las fases de la Luna.

Palabras clave: concepciones alternativas, educación primaria, astronomía

\section{ABSTRACT}

Our study is addressed to find misconceptions related to the Sun-Earth-Moon system, in the second and third cycle of primary school students (8-12 years old). A questionnaire with six open questions was used as instrument. The answers were analyzed and revealed that a high percentage of students surveyed have misconceptions about the Earth's orbit, the seasons and the Moon phases.

Key words: misconceptions, elementary education, astronomy

\section{INTRODUCCIÓN}

Numerosos autores han señalado que la formación de los maestros de primaria en ciencias es escasa (Camino, 1995) y con frecuencia presentan concepciones alternativas sobre diversos conocimientos en ciencias que deben enseñar al alumnado, por lo que estas ideas alternativas son transmitidas a niños y adolescentes, estableciendo una idea muy alejada a la científica, lo que apunta a la importancia de la formación de maestros.

En España, la astronomía se enseña a partir de educación primaria, en la asignatura de "Conocimiento del medio natural, social y cultural”, en sus tres ciclos. En el primero (6-7 años) se tratan: orientación de elementos del medio físico en relación con

\footnotetext{
“Universidad de Extremadura, España; flori@unex.es; flori.uex@gmail.com
} 
el Sol; percepción y descripción de algunos elementos y fenómenos naturales: la Luna, las estrellas y el Sol, el día y la noche. En segundo ciclo (8-9 ańos): los puntos cardinales; movimientos de la Tierra y fases de la Luna; las estaciones del año. Y, en tercer ciclo (10-11 años): el Universo, el Sistema Solar.

Las investigaciones sobre las concepciones alternativas de los estudiantes de educación primaria sobre temas de astronomía son escasas, aunque el asunto sea de gran importancia. Por el contrario, las realizadas con el alumnado de educación secundaria y docentes en formación y en activo son más abundantes. Por ello, el presente trabajo aborda el estudio (cuantitativo y cualitativo) sobre las ideas alternativas del alumnado de segundo y tercer ciclo de educación primaria, en concreto sobre el sistema Sol-Tierra, las causas de que haya veranos e inviernos y las fases de la Luna.

\section{FUNDAMENTACIÓN TEÓRICA}

El constructivismo concibe los conocimientos previos como esquemas que pueden ser variables entre el alumnado por la cultura familiar, las amistades, los medios de comunicación, que marcan la diferencia al respecto. Por ello las ideas previas son muy variadas según el momento, las circunstancias vividas, lo que crea cada uno, etc., y por esto, se dan diferentes opiniones sobre un mismo tema.

Así también la misma perspectiva teórica concibe la enseñanza y el aprendizaje de las ciencias como una construcción cognitiva que parte de un conocimiento previo (Novak, 1988; Posner, et al., 1982; Resnick, 1983; Driver, 1986 y 1988), por lo cual recomienda indagar sobre los que posee el alumnado, para poder abordar el aprendizaje de los nuevos contenidos. El constructivismo trata de averiguar lo que sabe el alumnado para transformar esas ideas (cambio conceptual) hacia las científicamente correctas.

Ausubel (1976) incorpora el concepto de aprendizaje significativo. Para este autor, aprender es sinónimo de comprender. Por ello, lo que se comprenda será lo que se aprenderá y recordará mejor, porque quedará integrado en nuestra estructura de conocimientos.

A su vez, Herrera (2009) comenta la exploración de qué, cuándo y cómo explorar y evaluar los conocimientos previos. 
En el qué explorar, recomienda: indagar sobre los conocimientos previos necesarios para poder abordar el aprendizaje de los nuevos contenidos y, en segundo lugar, los objetivos que tiene el maestro respecto al nuevo contenido. Sobre el cuándo, apunta que se ha de realizar siempre que el maestro lo considere necesario. Y en lo referente al cómo, destaca que existen infinidad de métodos y que el maestro utilizará el que considere mejor.

Por tanto, resulta fundamental para el profesorado no solo conocer las representaciones que posee el alumnado sobre lo que se le va a enseñar, sino también analizar el proceso de interacción entre el conocimiento nuevo y el que ya posee. De esta manera, no es tan importante el resultado final al que arriba el alumno como el proceso que le lleva a dar una determinada respuesta.

\section{Ideas previas: Sistema Sol-Tierra-Luna}

Las personas elaboramos nuestras propias ideas o concepciones para explicar el mundo que nos rodea (fenómenos naturales, cambios cotidianos). Estas ideas tienen cierto orden y organización y son de sentido común, ya que se construyen a partir del entorno. A la vez son universales y muy resistentes al cambio, por lo que pueden ser un obstáculo en la comprensión del conocimiento (Porta, 2007; Bello 2004). Estas ideas han sido denominadas de diversas formas: ideas previas, preconcepciones, concepciones alternativas, errores conceptuales, etc. En este estudio nos referiremos a estas como ideas o concepciones alternativas, pues consideramos que es la denominación que mejor refleja lo que son: ideas o concepciones que nos ayudan a explicar los fenómenos que observamos, pero no son científicamente correctas, por eso se denominan alternativas.

La manera en que estudiantes y maestros construyen los conceptos de astronomía resulta importante de cara al posterior estudio de las mismas. La mayoría de las investigaciones nos muestra que son muchas las concepciones alternativas que los estudiantes, tanto de enseñanzas primarias como superiores, tienen acerca de las relaciones del Sistema Sol-Tierra-Luna.

La mayoría de los estudios sobre los saberes de niños y adultos, así como de maestros y alumnado respecto a temas de astronomía se han enfocado a las estaciones del año, fases de la 
Luna, y el día y la noche (De Manuel, 1995; Kikas, 2004; Parker y Heywood, 1998; Vega, 2001). Estos estudios han encontrado concepciones alternativas en los maestros, similares a las identificadas en la población infantil.

Solbes y Palomar (2011) realizaron un estudio donde analizan las teorías y modelos más usados a lo largo de la historia de la ciencia para explicar el Sistema Solar y el Universo, donde se observa que las ideas o concepciones del profesorado, así como su enseñanza son muy importantes para el aprendizaje del alumnado, ya que este acepta las explicaciones por la autoridad que aquel representa, al igual que el libro de texto y, de la misma forma, las olvida. Además, se muestra cómo las dificultades de comprensión de la astronomía están relacionadas con una serie de factores, como el hecho de que la historia de la astronomía sea uno de los procesos más complejos en toda la historia de la ciencia, la ausencia de observaciones del cielo nocturno y diurno, el problema de las escalas, etc., por lo que una presentación de estos temas que siga el desarrollo histórico, puede contribuir a mejorar la enseñanza de los mismos y a superar algunas dificultades de los estudiantes.

A continuación haremos una revisión sobre algunos trabajos que estudian las concepciones alternativas en astronomía, centrándonos sobre todo en aquellos que han trabajado con alumnos de primaria y con maestros de primaria en formación. También, consideramos interesantes algunos trabajos donde se proponen métodos para superar estas concepciones alternativas.

Martín del Pozo et al., (2013), realizan un estudio sobre las ideas alternativas del alumnado de primaria (6-12 años) sobre la ciencia en general, centrándose, en la segunda parte de la investigación, en el "Sistema Sol-Tierra". Para ello, plantean multitud de cuestiones para conocer los niveles de conocimiento sobre este tema, la diversidad de ideas y la complejidad de las mismas. Dentro de los contenidos del sistema Sol-Tierra, se han investigado las ideas de percepción general acerca del sistema Sol-TierraLuna, el día y la noche, las estaciones, las fases de la Luna y los eclipses, donde los autores comprueban que, a lo largo de la etapa de educación primaria, se aprecia una mejora en la percepción del sistema Sol-Tierra-Luna desde un punto espacial, en los tamaños de los tres cuerpos y su posición entre sí y también en el 
conocimiento de los movimientos de los tres cuerpos. Respecto al día y la noche, en general, los alumnos establecen que la presencia de la Luna en el cielo es causa de las noches. Así también, las explicaciones del alumnado en referencia a las estaciones del año se fundamenta en la idea de la distancia de la Tierra al Sol y explican las fases de la Luna por la obstrucción de otros cuerpos (Sol, Tierra, nubes, planetas).

Santos Tena (2012) realizó un estudio con alumnos de sexto de primaria en un colegio público de educación primaria en España, con el objetivo de conocer las representaciones mentales que tienen acerca de la astronomía, concretamente sobre el Sistema Sol-Tierra, y las causas que hacen que haya veranos e inviernos. Para llevarlo a cabo se pasó un cuestionario elaborado a partir del diseñado por De Manuel (1995), con algunas modificaciones para adaptarlo al nivel académico del alumnado, en el que se analizó el pretest. Después, tras una intervención, se comparó el pretest, con el postest, para comprobar si esta ayuda a la superación de las concepciones alternativas. Los resultados fueron satisfactorios, ya que un porcentaje elevado de los estudiantes de sexto de primaria pudieron reestructurar sus concepciones y vencer sus ideas alternativas.

Kikas (2004) estudió en alumnos y maestros de primaria, las ideas alternativas sobre diversos temas de astronomía, como las estaciones del ańo. Pudo observar que maestros (56\%) y alumnos (72\%) poseían la idea alternativa de que las estaciones del año se deben a los cambios de la distancia entre el Sol y la Tierra.

El trabajo de Varela Losada et al., (2013) señala la persistencia de algunas concepciones alternativas sobre astronomía en el profesorado en formación. Para ello realizan una intervención educativa con actividades de simulación, identificando primero las ideas previas (pretest), para observar, en el postest, la eficacia de esa intervención. El estudio muestra que el alumnado presentó una gran cantidad de concepciones alternativas relacionadas con los aspectos astronómicos, como que las fases de la Luna son causadas porque esta entra y sale de la sombra de la Tierra o del Sol; el día y la noche son causados porque la Tierra se mueve alrededor del Sol; las diferentes estaciones son debidas a la distancia que varía entre el Sol y la Tierra. La intervención educativa realizada 
tuvo éxito ya que, en general, se observó una mejoría en los niveles de aciertos de casi todas estas cuestiones.

Bach y Franch (2004), a través de su estudio, llegaron a la conclusión de que los estudiantes de primero de magisterio poseen la idea de un modelo de órbita elíptica, con el Sol situado en uno de sus focos. Para explicar las estaciones, recurren a la concepción de la variación de las distancia Tierra-Sol. Mientras que para preguntas más complejas del Sistema Sol-Tierra, combinan las concepciones anteriores con otras (movimiento de rotación de la Tierra, inclinación del eje terrestre).

Con el estudio de Vega (2001) en relación con las nociones del día y la noche, se expuso que solo $55 \%$ de los maestros de primaria sostuvo la concepción científica, mientras la concepción alternativa más frecuente fue que la Tierra rota sobre sí misma (29\%), y a ambos lados se sitúan el Sol y la Luna, diametralmente opuestos.

Camino (1995) realizó una comparación antes y después de la implantación de una unidad didáctica, frente a las ideas previas de los estudiantes de magisterio sobre el día y la noche, las estaciones y las fases de la Luna. La intervención consistió en una serie de talleres donde los alumnos participaban de forma activa en el desarrollo y reflexión de las actividades, según la perspectiva constructivista del cambio conceptual. Ahí se pudo comprobar que las concepciones alternativas más frecuentes respecto al día y la noche eran que la Tierra rota sobre su eje sin trasladarse, ubicada en el centro entre la Luna y el Sol; los cambios en la distancia entre el Sol y la Tierra fue la respuesta que más se repitió para explicar el porqué de las estaciones del año. Después de los talleres se comprobó que los alumnos mejoraron en las explicaciones sobre los fenómenos astronómicos cuestionados, disminuyendo el porcentaje de quienes presentaban concepciones alternativas.

El estudio realizado por Cardenete (2011) propone la construcción de modelos para la explicación de los fenómenos que se producen como consecuencia de los movimientos de la Luna, la Tierra y de estas alrededor del Sol. Trabajan el día y la noche; las estaciones del año; las fases de la Luna y los eclipses de Sol y de Luna. Por ejemplo, para simular el día y la noche, colocan un proyector que simula al Sol, frente a un globo terráqueo que rota. Antes de realizar las simulaciones plantean una serie de preguntas sobre los fenómenos que van a trabajar. Respecto a la pregunta 
de por qué hay veranos e inviernos, sorprende la frecuencia de la respuesta "cuando estamos más cerca del Sol es verano y cuando estamos más lejos es invierno".

Piaget (1984) también estudió cómo abordan los niños la causa y la naturaleza de la noche, y diferencia cuatro fases de desarrollo. En la primera, la noche se explica de manera artificialista y finalista: los niños y las niñas de esta etapa piensan que la noche llega para poder dormir. La segunda tiene una explicación, ya que la noche aparece transportada por la oscuridad o por nubes, generalmente negras. En la tercera hay un cambio trascendente, la oscuridad o las nubes no son propiamente la noche, sino que se encargan de tapar el día y la luz solar. Y en la cuarta etapa, la noche resulta de la desaparición del Sol, lo que aporta evidencia de la dificultad de la enseñanza-aprendizaje de la astronomía. Con ello pone de manifiesto cómo las concepciones alternativas respecto a fenómenos observables son universales e incluso, aunque en menor grado, las detectadas en los alumnos de primaria, están presentes también en los maestros en formación. Algunos de estos trabajos ofrecen pautas e ideas que permiten detectar las ideas previas de los alumnos y la forma de abordarlas para llegar al cambio conceptual.

\section{Detección de las ideas previas}

Las investigaciones han puesto de manifiesto que las técnicas de enseñanza son más eficaces cuando, en primer lugar, se identifican las ideas de los alumnos. Bello (2004) apunta diferentes formas y estrategias para detectar las ideas alternativas de los alumnos, como plantear variadas situaciones en clase para que el niño deje traslucir sus ideas; escuchar las conversaciones que realizan con sus compańeros, así como observar y analizar los textos elaborados por el alumnado y sus representaciones tanto en dibujos, como en recursos esquemáticos y mapas conceptuales. También plantear un tema en coloquios, debates, torbellino de ideas, dibujos y, sin haber explicado nada, pedir opinión sobre el asunto que se trata, respuestas a preguntas planteadas del tema en cuestión, y a través de imágenes o fotos, de analogías. Son importantes las acciones que el docente realiza para conflictuar, desestabilizar, cuestionar a partir de lo cotidiano o creando situaciones que lo posibiliten. 
Las ideas alternativas tienen componentes de significado y sentido para los nińos, los cuales hacen referencia a sus intereses en el aprendizaje por lo que, en la medida que se conocen y se retoman, se atienden sus necesidades e intereses.

\section{PLANTEAMIENTO DEL PROBLEMA}

La importancia de estudiar las concepciones del alumnado de primaria sobre temas de astronomía y la escasez de trabajos acerca de ello justifican esta investigación.

El objetivo de este estudio es, pues, detectar concepciones alternativas relacionadas con la astronomía en un pequeño grupo de alumnos pertenecientes a un mismo centro educativo. Esperamos que los datos obtenidos con esta pequeña muestra confirmen los de otros estudios más grandes. Además, en este estudio se quiere mostrar la importancia de conocer las ideas alternativas tanto de los alumnos como de los propios docentes y ofrecer referencias de intervenciones didácticas para atender cada uno de los problemas abordados.

\section{METOdOLOGÍA DE INVESTIGACIÓN}

La metodología utilizada para desarrollar este estudio sigue un enfoque descriptivo-interpretativo. La muestra está formada por alumnos de segundo y tercer ciclo de primaria (9-12 años) de un colegio de educación infantil y primaria de la ciudad de Badajoz, España. No se tomó en cuenta el primer ciclo (alumnos de 6-8 años) por petición del centro.

En total, han participado 73 alumnos. En cuadro 1 se muestra el reparto de alumnos por curso:

CUADRO 1. Muestra por curso estudiado

\begin{tabular}{|c|l|}
\hline \multicolumn{1}{|c|}{ Curso } & \multicolumn{1}{|c|}{ Número de alumnado } \\
\hline $3^{\circ}$ Primaria $\left(2^{\circ}\right.$ Ciclo) & 21 alumnos/as \\
\hline $4^{\circ}$ Primaria $\left(2^{\circ}\right.$ Ciclo) & 23 alumnos/as \\
\hline $5^{\circ}$ Primaria $\left(3^{\circ}\right.$ Ciclo) & 11 alumnos/as \\
\hline $6^{\circ}$ Primaria $\left(3^{\circ}\right.$ Ciclo) & 18 alumnos/as \\
\hline
\end{tabular}


Los instrumentos para la recogida de datos han sido dos tipos de cuestionarios elaborados a partir del diseñado por De Manuel (1995), Santos Tena (2012), Bach y Franch (2004), Fernández y Peña (2007), entre otros, incluyendo algunas modificaciones para adaptarlo a los ciclos de primaria.

El cuestionario general (anexo), consta de seis preguntas, de las cuales a los alumnos de segundo ciclo se pasaron las siguientes:

¿Por qué pensáis que en verano hace más calor que en invierno?

¿Por qué creéis que en una parte del mundo es verano y en otra invierno? ¿Has oído hablar de la cara oculta de la Luna?, ¿a qué se debe?

¿Cuándo vemos a la Luna y de dónde viene su luz?

¿Por qué cambia de forma la Luna?

Para el tercer ciclo, además de estas preguntas, se añadieron dos más referentes a la trayectoria que sigue la Tierra alrededor del Sol y al porqué de los veranos e inviernos.

En el cuestionario se pregunta sobre aspectos astronómicos observables desde al ámbito cotidiano del alumno. Todos los conceptos tratados se abordan en estas etapas iniciales de enseñanza, en mayor o menor profundidad, aunque siempre se trabajan de una manera directa, presentando los hechos sin un cuestionamiento, por lo que consideramos interesante saber cuál es la justificación que aporta el alumno sobre los fenómenos astronómicos que puede observar en su día a día.

Las preguntas del cuestionario son sencillas, presentan situaciones cotidianas fácilmente identificadas por el alumno, bien en primera persona o por los medios de comunicación, en relación con la pregunta de la diferencia de estaciones en los hemisferios terrestres.

El hecho de realizar un cuestionario basándonos en preguntas formuladas en otros estudios se debe a que buscamos comparar los resultados de nuestra pequeña muestra con los de otras investigaciones, añadiendo, además, el hecho de realizar el cuestionario en dos ciclos y poder comparar cómo evolucionan las justificaciones de los alumnos respecto a los mismos fenómenos.

Por otro lado, el procedimiento llevado a cabo consta de varios pasos: en primer lugar, se pasó el cuestionario al segundo y al tercer ciclo de primaria, con el objetivo de averiguar las ideas que presenta el alumnado respecto al Sistema Sol-Tierra-Luna y 
las estaciones del año. A continuación, se categorizaron y codificaron todos los datos para tratarlos con el programa Microsoft Excel, 2010, y realizar un análisis no inferencial descriptivo de frecuencias. Finalmente, analizamos las respuestas obtenidas para abordar las conclusiones y la discusión.

Los cuestionarios fueron pasados por los tutores de cada curso. El tiempo que se dedicó a realizarlo fue de unos 30 a $35 \mathrm{mi}-$ nutos, aproximadamente.

\section{RESULTADOS Y DISCUSIÓN}

Analizaremos los resultados de cada una de la preguntas, haciendo una comparación en aquellas que son comunes a los dos ciclos.

\section{Pregunta 1}

Esta pregunta estaba presente en los cuestionarios pasados al tercer ciclo de primaria. En ella se presentan tres dibujos de la órbita que describe la Tierra alrededor del Sol; los alumnos debían seleccionar uno de ellos. La opción A se corresponde con una órbita elíptica con el Sol en uno de los focos. La opción B se corresponde con una órbita elíptica con el Sol en el centro. La opción C se corresponde con una órbita circular con el Sol en el centro.

En la gráfica 1 se muestran los porcentajes de elección de cada una de las opciones:

GRÁFICA 1. Porcentaje obtenido respecto a la opción elegida en la primera pregunta del cuestionario pasado al tercer ciclo de primaria

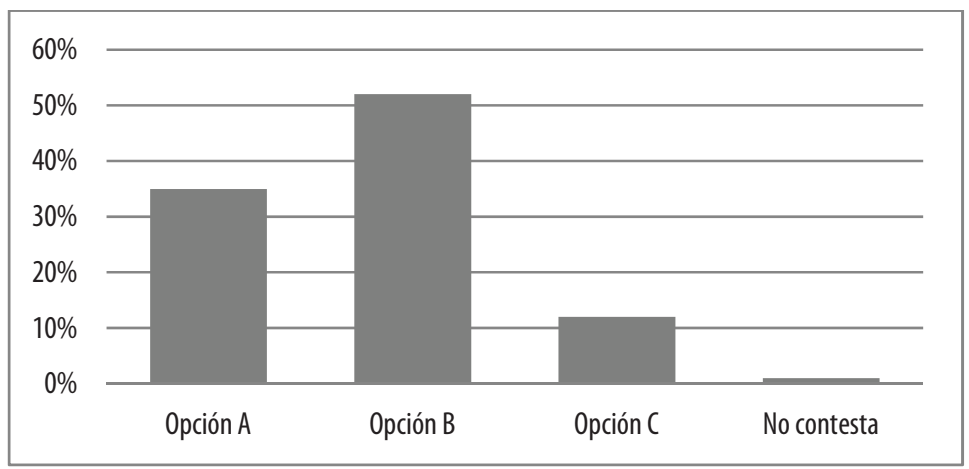


Como podemos observar, la elección de trayectorias elípticas A y B fueron las más frecuentes. La trayectoria circular (opción C) fue la elegida en menor rango. Es, por tanto, que 52\% del alumnado de tercer ciclo de primaria marcó la opción $\mathrm{B}$, considerando que la órbita terrestre es elíptica, con el Sol en el centro, seguida de la opción A, con 35\%, donde optan por la órbita terrestre elíptica, situando el Sol en uno de los focos; $12 \%$ cree que la órbita terrestre es circular, situando el Sol en el medio.

Estos resultados son prácticamente iguales que los obtenidos en el estudio realizado por De Manuel (1995), autor de este cuestionario, que con una muestra de 128 estudiantes de primaria, entre 12 y 13 años, obtuvo 35\% de la opción A, 55\% de la opción B y $10 \%$ de la C. En el estudio de Santos Tena (2012), 52\% del alumnado marcó la opción A (la correcta).

El mismo cuestionario fue utilizado en el estudio de Bach y Franch (2004), que se pasó a estudiantes de magisterio, con el objetivo de conocer sus concepciones alternativas a lo largo de nueve años. En total, se entrevistó a 732 estudiantes y se puede observar cómo prácticamente el conjunto de los alumnos opta por una órbita elíptica (opciones A y B), eligiendo correctamente más de $80 \%$ de los entrevistados durante este lapso el dibujo A. Con ello ratifican que la enseñanza previa al ingreso a la universidad consigue que la mayoría del alumnado tenga un modelo de órbita terrestre correcto.

\section{Preguntas 2 y 3}

Ambas se relacionan con la existencia de estaciones. En la primera se pregunta a los alumnos por qué piensan que hace más calor en verano y se les pide que realicen un dibujo donde representen la situación del Sol y la Tierra en ambas estaciones. Con ello se ha pretendido averiguar si el texto escrito y el dibujo se complementan y permiten explicar un modelo coherente que tenga correspondencia con el modelo científico para, en los casos en los que esta no existe, buscar dónde se encuentran las dificultades y, por lo tanto, con qué causa lo relacionan.

En relación con los dibujos, se ha analizado si los estudiantes eran capaces de representar la órbita terrestre, la inclinación del 
eje terrestre, el ángulo de los rayos solares en verano e invierno, la situación de la Tierra y el Sol en verano e invierno.

En la gráfica 2 se muestran las categorías obtenidas de la interpretación de los dibujos de los alumnos de tercer ciclo, así como el porcentaje que optó por cada una.

GRÁFICA 2. Justificación de la existencia de veranos e inviernos, de los cuestionarios pasados en el tercer ciclo de primaria

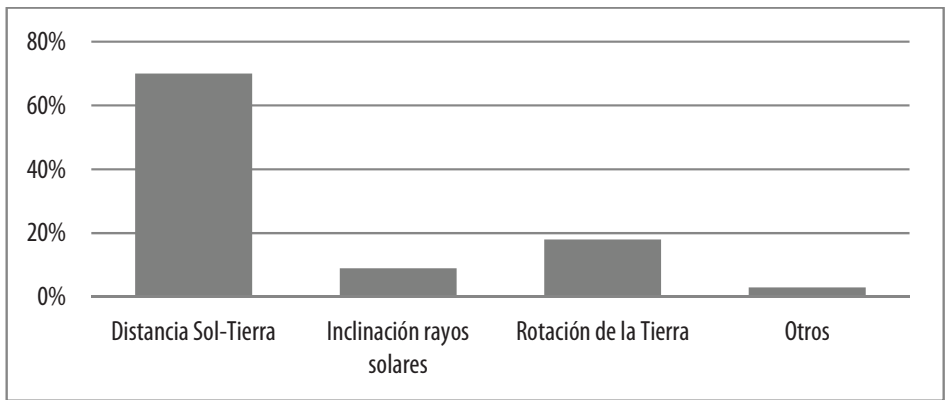

Analizados los dibujos, nos encontramos que 70\% de los estudiantes de quinto y sexto de primaria contestan que la existencia de veranos e inviernos se debe a la distancia entre el Sol y la Tierra, considerando que la Tierra se encuentra más cerca del Sol en verano y en invierno más lejos.

Nos llama la atención que en la pregunta anterior, referente a la órbita terrestre, más de $80 \%$ elige la opción de órbita elíptica, y en estos dibujos encontramos un alto porcentaje de órbita circular, por lo que no se relacionan o confunden un concepto y otro.

En la figura 1 se muestra un dibujo representativo de esta categoría, correspondiente a los alumnos de tercer ciclo.

FIGURA 1. Dibujo correspondiente a la categoría distancia Sol-Tierra

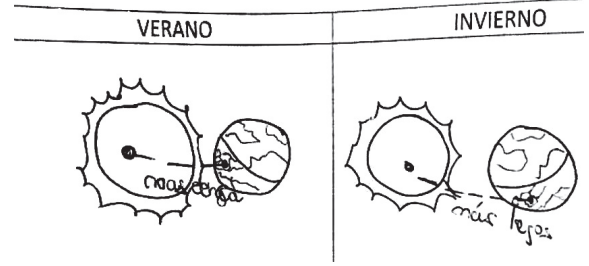


Tan solo tres estudiantes (10\%) han contestado correctamente, justificando este hecho en relación con la inclinación de los rayos del Sol. Efectivamente, las estaciones ocurren debido a que el eje de rotación terrestre tiene una inclinación aproximada de $23^{\circ}$ respecto a la normal del plano de la eclíptica, con lo que la inclinación de los rayos cambia, según la Tierra va girando alrededor del Sol. En verano, el hemisferio correspondiente es como si estuviera inclinado hacia el Sol, por lo cual los rayos solares llegan con mayor inclinación. Este dato representa que son muy pocos los alumnos que conocen la respuesta y por lo tanto no tienen claros conceptos básicos para contestar a preguntas acerca de la órbita, los movimientos de la Tierra o los hemisferios.

En la figura 2 se muestra un dibujo correspondiente a la categoría de inclinación de los rayos solares.

FIGURA 2. Dibujos correspondientes a la categoría Inclinación de rayos solares

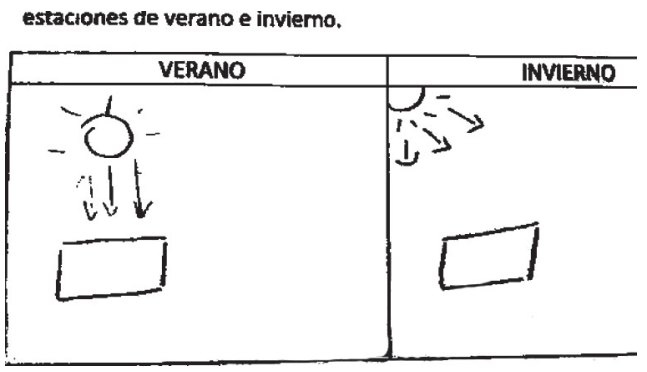

$17 \%$ de los estudiantes ha contestado que la existencia de verano e invierno se debe al movimiento de rotación terrestre. En la figura 3 observamos cómo el alumno señala a España, para diferenciar que en verano el continente se encuentra delante del Sol y en invierno lo sitúan detrás, colocando a América en la estación veraniega. Estos datos son similares a los obtenidos por Santos Tena (2012), donde 16\% de los alumnos de sexto de primaria utilizaron esta justificación. 
FIGURA 3. Dibujo correspondiente a la categoría Rotación de la Tierra taciones de verano e invierno.

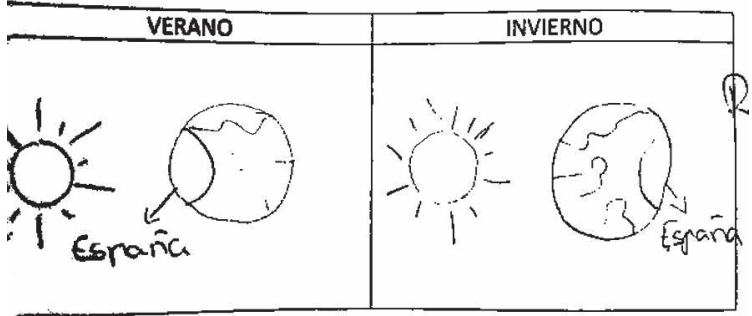

Respecto al análisis de las respuestas del alumnado de segundo ciclo de primaria, hemos obtenido el siguiente resultado que plasmamos en la gráfica 3 .

GRÁFICA 3. Justificación de la existencia de veranos e inviernos, de los cuestionarios pasados en el segundo ciclo de primaria

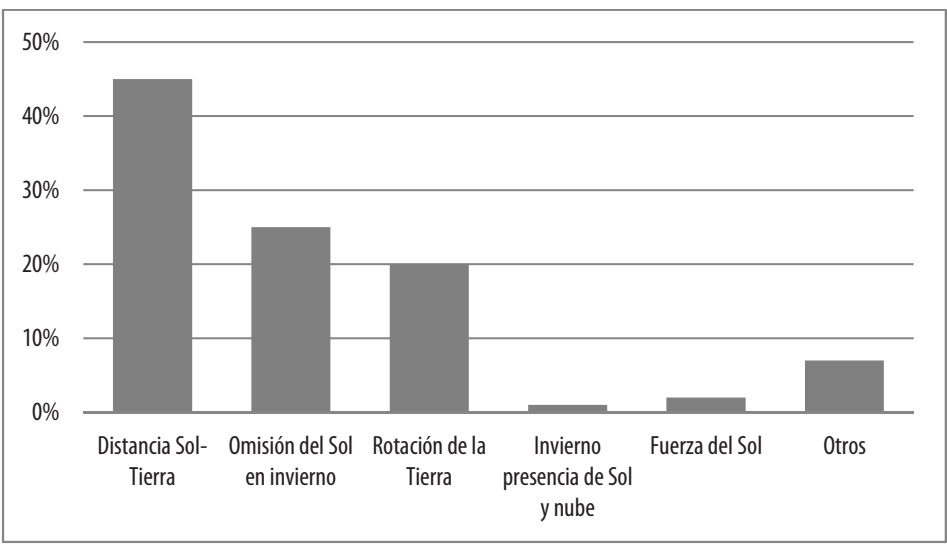

En esta gráfica puede verse cómo la distancia entre el Sol y la Tierra sigue siendo la respuesta que más eligen los estudiantes, ya que $45 \%$ del alumnado de tercero y cuarto de primaria justifican que los veranos e inviernos se deben a que en verano la Tierra se encuentra más cerca del Sol, y en invierno más lejos. Existen diferencias con los dibujos que encontramos en los cursos posteriores, ya que estos son más infantiles, representan al Sol animado y en ningún caso se representa la órbita. En la mayoría de los 
casos realizan un paisaje típico de cada estación, plasmando la distancia entre el Sol y la Tierra. También, representan la Tierra, el Sol y la Luna y la distancia entre ellas en verano e invierno. En otros casos, ańaden rayos solares, pero con una nota aclaratoria comentan que es debido a la distancia entre ellos. Lo mismo que en el caso anterior, ninguno de los estudiantes consideró que la Tierra está más lejos del Sol en verano.

En la figura 4 se muestra un dibujo representativo de la categoría distancia Sol-Tierra.

FIGURA 4. Dibujo correspondiente a la categoría distancia Sol-Tierra

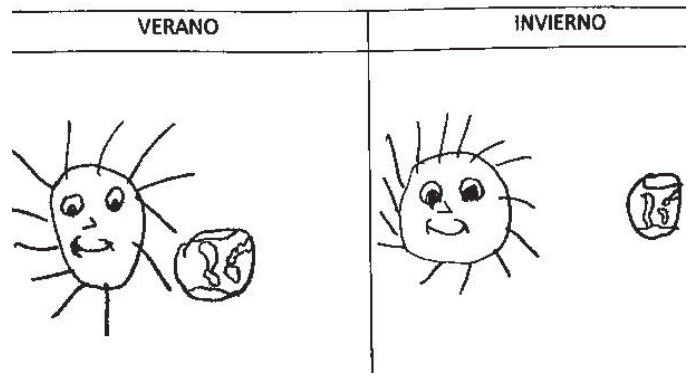

Por otro lado, 25\% del alumnado no dibuja el Sol en invierno, mientras que en verano lo dibujan claramente. No sabíamos por qué omitían el Sol, por ello preguntamos para poder justificar la respuesta. La mayoría de los estudiantes lo justificó diciendo que, "en invierno apenas sale el Sol, casi siempre hay nubes, mientras que en verano se encuentra todo el tiempo el Sol". Es por ello que la siguiente pregunta fue si, por lo tanto, en invierno el Sol se esconde y en verano aparece, a lo que contestaron que “¡así es!, en invierno el Sol se esconde más, apenas aparece por que tiene frío, y en verano, como hace calor, aparece". Con esta aclaración podemos observar que hay estudiantes a los que les falta interiorizar ciertos conceptos para aclarar este suceso, tales como la órbita terrestre, los movimientos de rotación y traslación de la Tierra y la inclinación del eje terrestre.

También los alumnos del segundo ciclo han recurrido al movimiento de rotación para la justificación del verano y el invierno $(20 \%)$. En este caso comprobamos que el libro de texto y las clases 
de astronomía a través de la asignatura de Ciencias Sociales, hacen mucho hincapié en el aprendizaje de los movimientos de rotación y traslación de la Tierra con el Sol. Tanto es así que, para la explicación del tema, el alumnado, con la ayuda de la maestra, pintó tres bolas de corcho de diferentes tamaños (el Sol más grande, la Tierra un poco más pequeña y la Luna más pequeña aún) y recreó cómo sería el movimiento individual de cada uno y entre ellos.

Encontramos más variables de respuestas sobre esta cuestión, que no se habían mencionado en el tercer ciclo, como la fuerza del Sol, con ilustraciones con un Sol más grande en verano y más pequeño en invierno. Un alumno representó en invierno la presencia de Sol y nubes, significando en comentarios aclaratorios, que "en esta estación, a causa de las nubes, surge el invierno; todo lo contrario que en verano, que no hay muestra de nubes y por ello calienta más el Sol".

Si comparamos estos resultados con la pregunta del cuestionario pasado por De Manuel (1995), comprobamos que 61\% del alumnado de entre 12-13 años, justificó la existencia de estaciones con base en la distancia entre el Sol y la Tierra, resultado muy parecido al de nuestro estudio (45\% en el segundo ciclo y $70 \%$ en el tercer ciclo de primaria). Con esto constatamos que, a pesar de la diferencia de años entre ambos estudios, las concepciones alternativas de los alumnos son las mismas.

Respecto a esta misma cuestión establecida a maestros de primaria (Fernández y Peña, 2007), 86\% presentó concepciones alternativas o no aportó suficiente información. Las concepciones más frecuentes identificadas se basan en la variación de la distancia entre la Tierra y el Sol. Destacan que en verano estamos más cerca del Sol y por eso hace más calor y en invierno más lejos y por eso hace más frío. En esta investigación, como en otras donde se estudian las causas de este fenómeno en maestros de primaria (Parker y Heywood, 1998; Bach y Franch, 2004); Vega, 2007; Solbes y Palomar, 2011), ponen de manifiesto que la mayoría de los maestros encuestados no son conscientes de que poseen concepciones alternativas sobre estos temas.

De acuerdo con De Manuel (1995), justificar los veranos e inviernos demuestra las dificultades que entraña el tema en cuestión, además de ser una concepción que nace de la experiencia y 
del sentido común y que, por lo tanto, resulta estar muy arraigada, lo que abunda en la necesidad de detectar, en primer lugar, las ideas previas, para poder llegar a un cambio conceptual, tanto en maestros como en estudiantes, como una tarea principal que nos permitirá la alfabetización en ciencias.

\section{Preguntas 4 y 5}

Estas preguntas están relacionadas con la Luna y se plantearon tanto al tercer como al segundo ciclo de primaria.

Comenzando por la primera, se sabe que la cara oculta de la Luna es la que no es observable desde la Tierra. Por tanto, nos preguntamos, ¿̨por qué siempre vemos la misma cara? Esto es debido a que la Luna rota sobre sí misma al mismo tiempo que se traslada alrededor de la Tierra, es decir, su periodo de rotación es igual al de traslación, lo que origina que siempre veamos la misma cara.

En la gráfica 4 se muestran los resultados de las respuestas de los alumnos del tercer ciclo y en la 5 las de los alumnos del segundo ciclo de primaria.

GRÁFICA 4. Respuestas sobre qué es la cara oculta de la Luna, del tercer ciclo de primaria

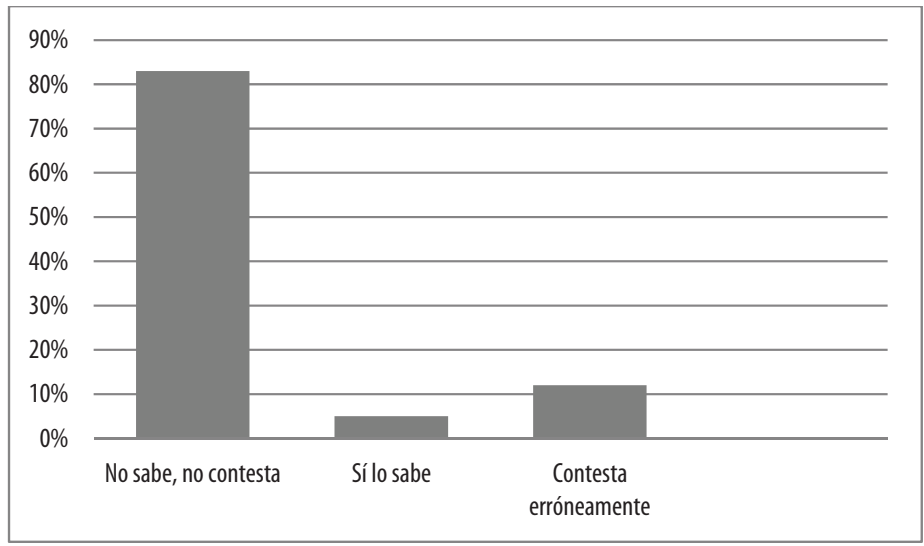


GRÁFICA 5. Respuestas del segundo ciclo de primaria, sobre que es la cara oculta de la Luna

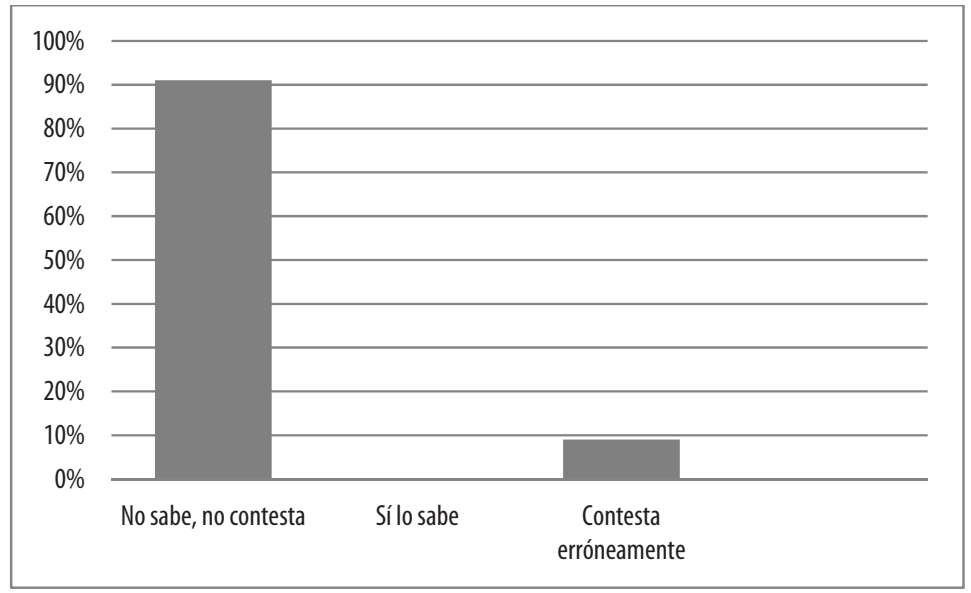

Respecto al tercer ciclo: en esta pregunta se puede observar que $83 \%$ del alumnado no sabe qué es la cara oculta de la Luna. Tan solo un alumno conocía este fenómeno y contestó de la siguiente forma: “... la Luna gira sobre sí misma al mismo tiempo que se traslada alrededor de la Tierra, por eso siempre se ve la misma cara".

El resto de los estudiantes (13\%) de tercer ciclo dan otras explicaciones respecto a la pregunta de la cara oculta de la Luna, como, "Sí, se debe al Sol"; "Se debe a que el Sol no alumbra esa parte"; "Porque cambia según la luz dél Sol".

Respecto al segundo ciclo de primaria, observamos que no hay gran diferencia con respecto al tercer ciclo ya que $91 \%$ de la muestra no contestó la pregunta.

El restante 9\% la justificó contestando, "Sí, he oído hablar de la cara oculta de la Luna, es porque esa parte de la Luna le da la sombra de la Tierra", "Porque la Luna está llena y está blanca".

Con respecto a otros estudios que han trabajado este concepto, Parker y Heywood (1998) afirman que es difícil comprender que la Luna muestre siempre la misma cara. Esto se debe a que es necesario situarse en un marco de referencia fuera del sistema Tierra-Luna para poder ver la rotación de la Luna sobre su eje. Para explicarlo, proponen una actividad para facilitar la com- 
prensión de este hecho, en la que un alumno va girando alrededor de otro que representa a la Tierra. El alumno que se mueve mantiene el mismo periodo de rotación y traslación, de tal forma que siempre estará dando la cara al alumno que hace de Tierra.

Kriner (2004) analiza los libros de texto de primaria, para detectar insuficiencias en las explicaciones relacionadas con astronomía. Respecto a la cara oculta de la Luna, destaca una descripción que aparece en un libro de texto, en el que dice:

la Luna efectúa, además, otros dos movimientos: de rotación sobre su eje, y casi en el mismo tiempo el de traslación alrededor de la Tierra en 29 días, 12 horas y 44 minutos. Como consecuencia de esto siempre muestra a los habitantes de nuestro planeta la misma cara.

Kriner comenta que las afirmaciones del texto son correctas (los movimientos de rotación y traslación de la Luna tienen el mismo periodo y esto causa que se vea la misma cara) pero la duración que menciona no lo es.

Respecto a los diferentes estudios citados, como en el nuestro, podemos decir que la concepción de los estudiantes respecto a la cara oculta de la Luna es nula, ya que un porcentaje alto desconoce lo que es.

La otra pregunta relacionada con la Luna es: ¿¿De dónde viene la luz de la Luna?

La Luna no emite luz propia, sino que refleja la luz del Sol, mostrando sus características fases durante cada órbita alrededor de la Tierra, que dura en torno a 28 días; es decir, si nos situamos en una habitación oscura, no se puede ver nada de lo que hay en ella, pero si se enciende una luz se puede observar todos los objetos que están iluminados por ella, pues ellos reflejan la luz que reciben. Esto mismo le ocurre a la Luna, ya que su superficie refleja la luz solar. Esta pregunta se pasó al alumnado de segundo y tercer ciclo de primaria.

En las gráficas 6 (tercer ciclo) y 7 (segundo ciclo) se muestran las categorías en las que se englobaron sus justificaciones y el porcentaje que obtuvo cada una. 
GRÁFICA 6. Respuestas de los estudiantes de tercer y segundo ciclos de primaria, sobre la pregunta, ¿de dónde viene la luz de la Luna?

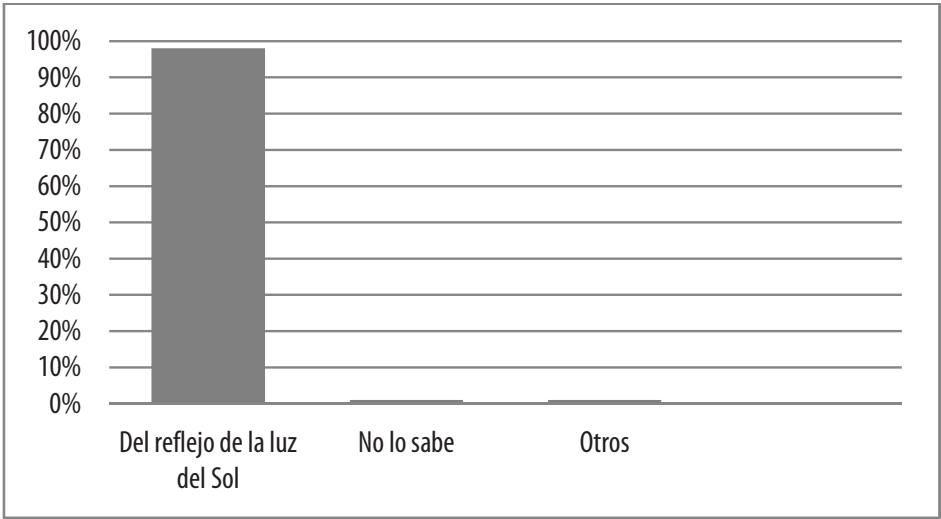

Como podemos observar, $97 \%$ de los estudiantes responde correctamente a esta cuestión. Resulta un concepto que está bastante afianzado en las ideas del alumnado.

En el estudio realizado por Cardenete (2011), se pasó un cuestionario con preguntas relacionadas con el Sistema Sol-Tierra-Luna, para conocer las ideas previas de los estudiantes. Entre ellas, se preguntó, ¿̇tiene la Luna luz propia? Para aclarar este concepto, este autor propone que se utilice una pelota y se mire mientras que se enciende y se apaga una linterna.

Tanto Cardenete (2011) como Kriner (2004) coinciden en que los alumnos comprenden mejor el concepto de reflexión de la luz por parte de la Luna, que las fases de la Luna.

La última pregunta, ¿Cuándo vemos a la Luna?, está relacionada con las cuestiones del día y la noche. En realidad, podemos ver a la Luna tanto de día como de noche, dependiendo de la fase en que esta se encuentre.

La comprensión de este concepto resulta muy complicada, ya que al verse mejor la Luna de noche que de día, la mayoría de los estudiantes y adultos se confunden y establecen, alternativamente, que de día la Luna desaparece y de noche aparece (Kriner, 2004; Cardenete, 2011). 
En la gráfica 7 se muestran los totales obtenidos después de analizar las respuestas de los alumnos del tercer y segundo ciclo.

GRAFICA 7. Respuestas de estudiantes de segundo y tercer ciclo de primaria a la pregunta ¿cuándo vemos a la Luna?

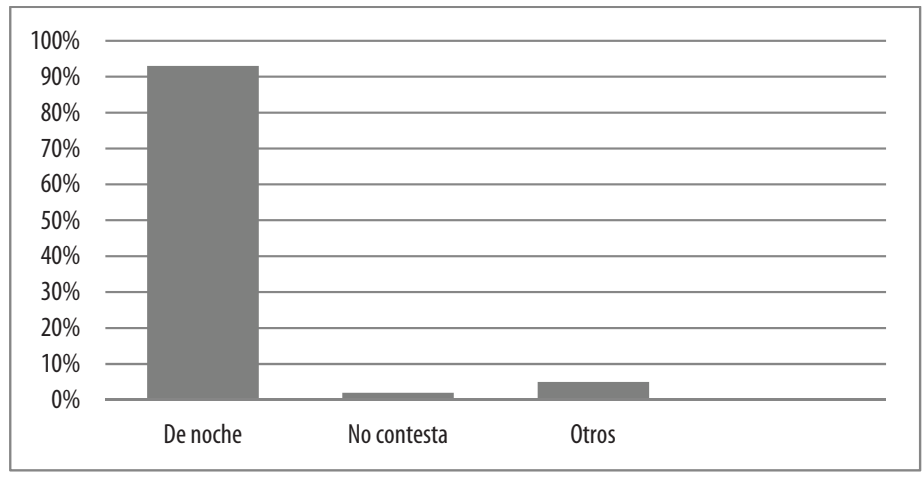

Según los resultados obtenidos en esta pregunta, 93\% del alumnado de segundo y tercer ciclo, opina que "la Luna se ve de noche", justificando que "durante el día se encuentra el Sol y ya es por la noche cuando aparece la Luna". Esta justificación no es correcta, ya que no se cumple durante todas las fases de la Luna. Por ejemplo, cuando la Luna se encuentra en su fase decreciente, la mayor parte del tiempo se ve durante el día.

Como hemos dicho antes, la Luna es un cuerpo opaco que refleja la luz que recibe del Sol y, por lo tanto, solo podemos ver las zonas que ilumina la estrella solar. Esto no quiere decir que la Luna desaparezca, como piensa la gran mayoría de niños y adultos, sino todo lo contrario; la Luna siempre está y puede verse también por el día, aunque no lo apreciemos tan nítidamente como por la noche, debido a la luz solar.

Comparando nuestros resultados con los obtenidos por Camino (1995), comprobamos que existen similitudes considerables, a pesar de que la muestra de su trabajo fue diferente, concretamente con maestros. En el estudio encontraron que $72 \%$ postula que la respuesta a cuándo se ve la luna, es por la noche. 
Además, este concepto se relaciona con el fenómeno del día y de la noche, pues los estudios de Camino (1995), Vega (2001) y Fernández y Peña (2007) obtuvieron que la concepción alternativa más frecuente, corresponde a la idea de que la Tierra rota sobre sí misma en el "centro", con respecto al Sol y la Luna, situados estos de forma opuesta. Por ello se confunde con que hay día y noche, debido al movimiento de rotación de la Tierra. Así, la Tierra gira sobre sí misma de Oeste a Este y tarda 24 horas, es decir, un día completo en realizar este movimiento. Debido a esto, a la rotación, la mitad de la Tierra permanece iluminada por el Sol y la otra mitad permanece a oscuras, siendo de este modo, cuando aparecen los periodos del día y la noche.

\section{Pregunta 6}

El conocimiento y aprendizaje de las fases de la Luna resultan de gran complicación tanto para nińos como para adultos, tal y como hemos visto en investigaciones de Kriner (2004), Vega Navarro (2007), Fernández Nistal y Peña Boone (2007), entre otros. Esta complicación de enseñanza y aprendizaje se debe al alto grado de abstracción de los conceptos y al conocimiento espacial que requieren.

Las fases de la Luna son las diferentes iluminaciones que presenta nuestro satélite en el curso de un mes lunar. Según la posición de la Luna, la Tierra y el Sol, se ve iluminada una mayor o menor porción de la cara visible de la Luna. Es decir, las distintas porciones iluminadas que vemos de ella, las llamadas fases lunares, dependen de la posición de la Luna y de la Tierra con respecto al Sol.

En las siguientes gráficas, se muestran los resultados obtenidos para las justificaciones del tercer ciclo (gráfica 8) y del segundo ciclo (gráfica 9). Como podemos observar, ningún estudiante de ambos ciclos contestó correctamente

En cuanto a las justificaciones de los alumnos de tercer ciclo, la que más se repite es "porque la Luna tiene diversas formas". Estos alumnos de tercer ciclo saben que la Luna cambia, pero no conocen cuál es el motivo científico de por qué se produce ese cambio. 
CONCEPCIONES ALTERNATIVAS DE ALUMNOS DE SEGUNDO Y TERCER CICLO DE PRIMARIA...

GRAFICA 8. Respuestas de estudiantes de tercer ciclo de primaria sobre la pregunta ¿Por qué cambia de forma la Luna?

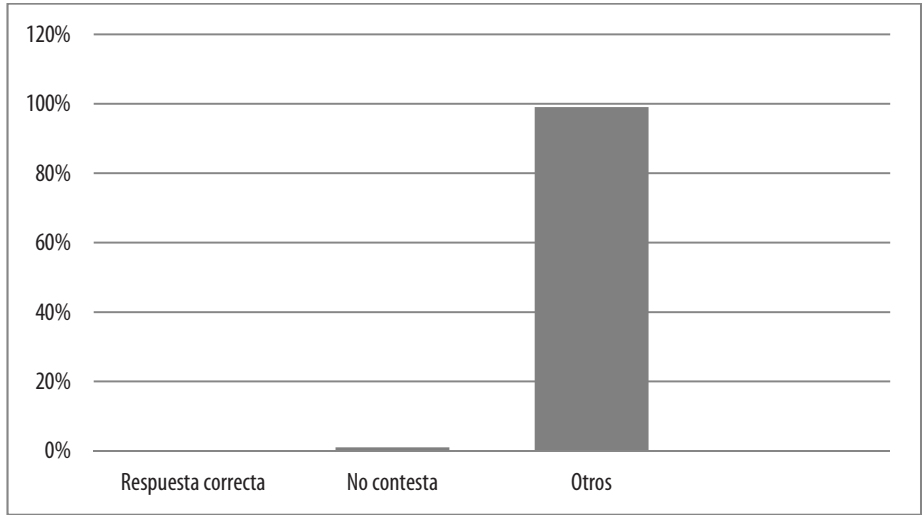

GRÁFICA 9. Justificación de los estudiantes de segundo ciclo de primaria a la pregunta ¿Por qué cambia de forma la Luna?

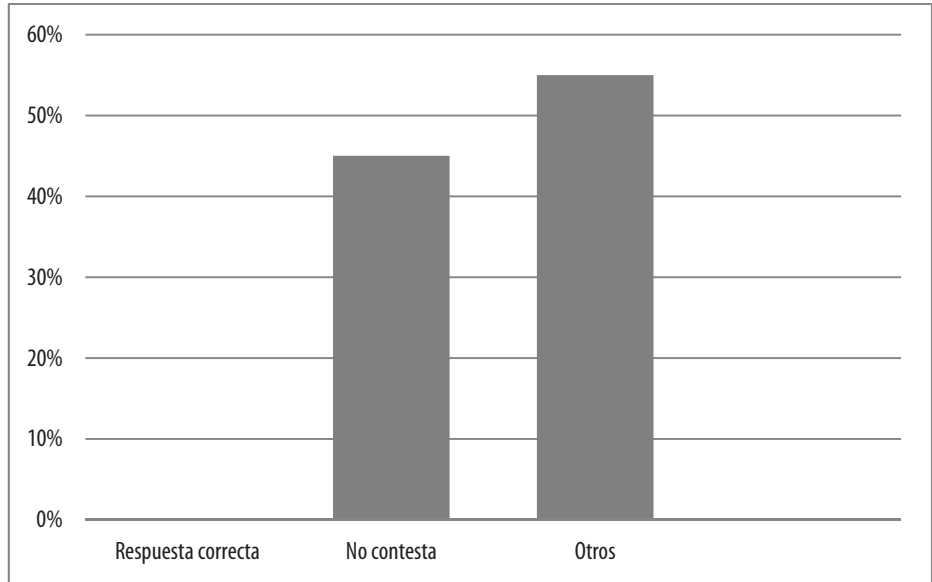

En segundo ciclo hubo un gran porcentaje de alumnos que dejó en blanco la pregunta (46\%). Esto puede deberse a que el alumnado de tercer ciclo posee más conocimientos debido tanto a su experiencia como a lo ya aprendido en cursos anteriores. De entre la justificaciones dadas por los alumnos que contestaron la pregunta (54\%), la que más se repite es "porque la Luna gira alrededor de la Tierra”. 
Según el concepto de las fases de la Luna, Piaget (1984) diferenció tres etapas evolutivas de los niños respecto a este concepto. La primera etapa, de total artificialismo, los cuartos de Luna, como trozos de Luna a la que alguien ha partido en dos. La segunda etapa, de artificialismo mitigado, porque se entiende que la Luna puede autoseccionarse o ser cortada por el viento. En la tercera etapa, aparecen ya explicaciones más naturales, es decir, la Luna desaparece parcialmente a causa de su propio movimiento o porque vienen nubes para taparla.

\section{CONCLUSIONES}

Los resultados obtenidos en nuestro estudio son coherentes con los objetivos marcados.

En la pregunta 1, la mayoría de los alumnos del estudio identifica una trayectoria elíptica de la órbita terrestre, situando al Sol en el centro de forma errónea, por lo que las enseñanzas recibidas con anterioridad y las concepciones que se tienen al respecto no son claras y deben abordarse de nuevo, para que se produzca un cambio conceptual.

Respecto a las preguntas 2 y 3 observamos que $70 \%$ del alumnado de tercer ciclo y $45 \%$ de segundo justifican la existencia de verano e invierno como consecuencia de la distancia entre la Tierra y el Sol. La mayoría de los alumnos del estudio posee la concepción alternativa de que las estaciones se deben a esta distancia. De nuevo, habrá que abordar esta temática de forma diferente a la usada para producir el cambio conceptual hacia la concepción científicamente correcta.

En las preguntas 4 y 5, 83\% del alumnado de tercer ciclo y $91 \%$ del de segundo, no sabe qué es la cara oculta de la Luna. Sin embargo, casi todos los alumnos del presente estudio conocen que la luz de la Luna proviene del Sol. Con respecto a cuándo vemos la Luna, prácticamente todos los alumnos argumentan que la Luna solo se ve por la noche.

Por último, en la pregunta 6 , vemos cómo $100 \%$ de los estudiantes de segundo y tercer ciclos de primaria no saben por qué cambia de forma la Luna, tema relacionado con las fases de esta. Aunque hemos encontrado limitaciones, una de ellas, el reducido 
número de integrantes de la muestra y que solo se ha estudiado en un único centro o que no se ha podido hacer una intervención didáctica, nos resta proponer posibles líneas de investigación derivadas de este trabajo, como investigar de forma cualitativa la metodología empleada por los maestros en referencia al aprendizaje de estos conceptos y realizar una intervención didáctica sobre el Sistema Sol-Tierra-Luna, tanto con estudiantes como con adultos, para comprobar en qué medida una acción educativa de este tipo ayuda a la superación de las concepciones alternativas, sobre todo entre el cuerpo docente.

\section{REFERENCIAS BIBLIOGRÁFICAS}

Atwood, R. K. "Preservice elementary teachers' conceptions of what causes night and day", en School Science and Mathematics, 95, 1995, pp. 290-294.

Ausubel, D. Psicología educativa. Un punto de vista cognoscitivo, México, Trillas, 1976.

Bach, J. y Franch, J. "La enseñanza del Sistema Sol-Tierra desde la perspectiva de las ideas previas", en Revista de las Ciencias de la Tierra, 12, 2004, pp. 302-312.

Baxter, J. "Children's understanding of familiar astronomical events", en International Journal of Science Education, 11, 1989, pp. 502-513.

Bello, S. "Ideas previas y cambio conceptual", en Educación Quimica, 15, 2004, pp. 210-217.

Camino, N. (1995). "Ideas previas y cambio conceptual en astronomía. Un estudio con maestros de primaria sobre el día y la noche, las estaciones y las fases de la Luna", en Enseñanza de la ciencias, 13, 1995, pp. 81-96.

Cardenete, S. "Sol, Tierra y Luna. Movimientos relativos y sus consecuencias", en Revista Eureka sobre enseñanza y divulgación de las ciencias, 8, 2011, pp. 512-518.

De Manuel, J. “¿Por qué hay veranos e inviernos? Representaciones de estudiantes (12-18) y de futuros maestros sobre algunos aspectos del modelo Sol-Tierra", en Enseñanza de las Ciencias, 13, 1995, pp. 227-236. 
Driver, R. "Psicología cognoscitiva y esquemas conceptuales de los alumnos", en Enseñanza de las Ciencias, 4, 1986, pp. 3-15.

Driver, R. "Un enfoque constructivista para el desarrollo del currículo en ciencias", en Enseñanza de las Ciencias, 6, 1988, pp. 109-120.

Fernández, M. T. y Peña, S. H. "Concepciones de los maestros de primaria sobre el día y la noche y las estaciones del año", en Revista Latinoamericana de Estudios Educativos, vol. XXXVII, 2007, pp. 2237-2246.

Herrera, A. M. "El constructivismo en el aula", en Innovación y Experiencias Educativas, 14, 2009, pp. 1-10.

Kikas, E. "Teachers' conceptions and misconceptions concerning three natural phenomena", en Journal of Research in Science Teaching, 41, 2004, pp. 432-448.

Kriner, A. "Las fases de la Luna, ¿Cómo y cuándo enseñarlas?", en Revista Ciência y Educaçao, 10, 2004, pp. 111-120.

Martín Del Pozo, R., Arillo, M. A., Ezquerra, A., Fernández, P., Galán, P., García, E., González, M., De Juanas, A., Reyero, C. y San Martín, C. Las ideas "científicas" de los alumnos y alumnas de primaria: tareas, dibujos y textos, Universidad Complutense de Madrid, Madrid, España, 2013.

Novak, J. D. Teoría y práctica de la educación, Madrid, Alianza, 1982.

Parker, J. y Heywood, D. "The earth and beyond: developing primary teacher's understanding of astronomical events", en International Journal of Science Education, 20, 1998, pp. 503-519.

Piaget, J. La representación del mundo en el niño, Barcelona, Morata, 1984.

Porta, S. "Las ideas previas y las situaciones de enseñanza", en Quehacer Educativo, 2007, pp. 146-149.

Posner, G. J., Strike, K. A., Hewson, P. W. y Gertzog, W. A. "Accommodation of a scientific conception: towards a theory of conceptual change", en Science Education, 66, 1982, pp. 211-227.

Resnick, L. B. "Mathematics and Science Learning: a new conception", en Science, 20, 1983, pp. 477-478. 
Santos Tena, M. I. "Evolución de las concepciones alternativas de alumnos del tercer ciclo de educación primaria sobre el sistema Sol-Tierra y las causas que hacen que haya veranos e inviernos", en Memoria para optar al Titulo del Máster oficial en investigación y aprendizaje de las ciencias experimentales, sociales y matemáticas, Facultad de Educación, Universidad de Extremadura, Badajoz, España, 2012.

Solbes, J. y Palomar, R. "¿Por qué resulta tan difícil la compresión de la astronomía a los estudiantes?”, en Revista de Didáctica de las Ciencias Experimentales y Sociales, 25, 2011, pp. 187-211.

Trumper, R. "The need for change in elementary school teacher training. A cross-college age study of future teachers' conceptions of basic astronomy concept", en Teaching o Teacher Education, 19, 2003, pp. 309-323.

Varela Losada, M. M., Pérez Rodríguez, U., Serralé Marzoa, J. F. y Arias Correa, A. "Evolución de las concepciones sobre astronomía de profesorado en formación tras una intervención educativa con actividad desde simulación”, en $I X$ Congreso Internacional sobre Investigación en Didáctica de las Ciencias, 2013, pp. 3612-3617.

Vega, A. "Tenerife tiene seguro de Sol (y de Luna). Representaciones del profesorado de primaria acerca del día y la noche", en Enseñanza de las Ciencias, 19, 2001, pp. 31-44.

Vega Navarro, A. "Ideas, conocimientos y teorías de niños y adultos sobre las relaciones Sol-Tierra-Luna. Estado actual de las investigaciones", en Revista de Educación, 342, 2007, pp. 475-500. 


\section{ANEXO}

1. Como ya sabemos, la Tierra gira alrededor del Sol mediante el movimiento de traslación. ¿Cuál de estos tres dibujos se aproxima más a la trayectoria que sigue la Tierra alrededor del Sol? Pon un círculo en la letra que consideres correcta.

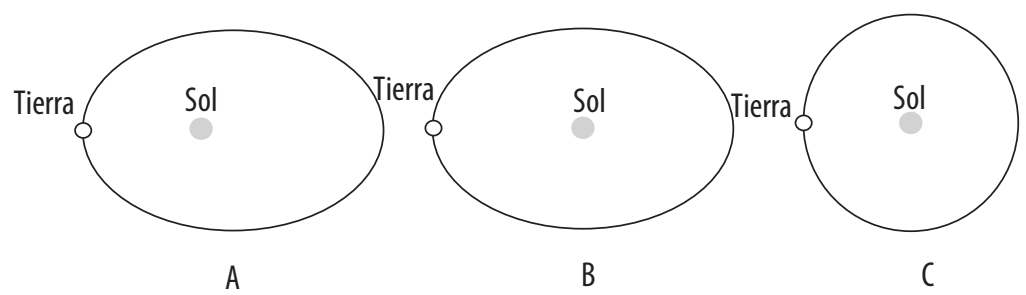

2. ¿Por qué pensáis que en verano hace más calor que en invierno? Realiza un dibujo donde representes la situación del Sol y la Tierra en las estaciones de verano e invierno.

3. ¿Por qué creéis que en una parte del mundo es verano y en otro invierno?

4. ¿Has oído hablar de la cara oculta de la Luna? ¿A qué se debe?

5. ¿Cuándo vemos a la Luna y de dónde viene su luz?

6. Cuando miramos al cielo de noche, no siempre vemos a la Luna, y cuando se ve, no siempre tiene la misma forma. ¿Por qué cambia de forma la Luna? 